\title{
Epigenetic Changes of FKBP5 as a Link Connecting Genetic and Environmental Risk Factors with Structural and Functional Brain Changes in Major Depression
}

\author{
Leonardo Tozzi*, I,2, Chloe Farrell', Linda Booij ${ }^{3,4}$, Kelly Doolin', Zsofia Nemoda ${ }^{5}$, Moshe Szyf \\ Florence B Pomares ${ }^{3,4}$, Julian Chiarella ${ }^{3,4}$, Veronica O'Keane' and Thomas Frodl ${ }^{1,2}$ \\ 'Department of Psychiatry, Trinity College School of Medicine and Trinity College Institute of Neuroscience, Dublin, Ireland; ${ }^{2}$ Department of \\ Psychiatry, Otto von Guericke University Magdeburg, Magdeburg, Germany; ${ }^{3}$ Department of Psychology, Concordia University, Montreal, Canada; \\ ${ }^{4} \mathrm{CHU}$ Sainte-Justine Hospital Research Centre, University of Montreal, Montreal, Canada; ${ }^{5}$ Department of Pharmacology and Therapeutics, McGill \\ University, Montreal, Canada
}

\begin{abstract}
The gene for the glucocorticoid receptor regulator FK506 binding protein 5 (FKBP5) plays a role for risk, response to treatment, and changes in brain areas in major depressive disorder (MDD). Chronic stress is associated with lower methylation of FKBP5. Our aim was to investigate whether methylation of FKBP5 reflected exposure to childhood adversity in MDD and controls and whether it was associated with structure and function of emotional processing regions. FKBP5 intron 7 GR response element region methylation and rs 1360780 allelic status were assessed from whole blood in 56 MDD adults and 50 controls. Using magnetic resonance imaging, we assessed gray matter concentration of selected areas and their function during valence recognition of emotional images. Childhood adversity was investigated using the Childhood Trauma Questionnaire. In MDD patients carrying the high-risk T allele of rs I 360780, lower methylation of FKBP5 was predicted by childhood adversity $(F=4.95, p=0.04)$. In all participants, lower FKBP5 intron methylation levels were associated with reduced gray matter concentration in the inferior frontal orbital gyrus bilaterally (Wald chi-square $=11.93, p_{F D R}<0.01$ ) and, in MDD, with its bilaterally higher activation during valence recognition (Wald chi-square $=5.58, p=0.02$ ). Activation of this region, regardless of side, was found to be lower in MDD compared to controls (Wald chi-square $=3.88, p=0.049$ ) and to be inversely correlated with depression severity (Wald chi-square $=4.65, p=0.03$ ). Our findings support the hypothesis that, in genetically predisposed individuals carrying a high-risk variant of the gene, childhood maltreatment might induce demethylation of FKBP5. This is in turn associated with structural and functional changes in the inferior frontal orbital gyrus, a relevant area for the clinical symptoms of MDD.

Neuropsychopharmacology (20 I8) 43, I | 38-I I45; doi: I 0.1038/npp.20 I7.290; published online 20 December 2017
\end{abstract}

\section{INTRODUCTION}

Many studies investigating the pathogenesis of major depressive disorder (MDD) have focused on dysregulation of the hypothalamic-pituitary-adrenal (HPA) axis and inflammation as key elements of the condition (Miller and Raison, 2016). Compatible with this hypothesis, many genes involved in HPA axis regulation have been examined to assess risk of developing MDD and as potential predictors of antidepressant treatment response (de Kloet et al, 2005).

The gene encoding the FK506 binding protein 5 (FKBP5), located on chromosome 6p21.31, is involved in steroid receptor sensitivity regulation creating an intracellular negative feedback loop (Scharf et al, 2011, p 20; Binder,

*Correspondence: Dr L Tozzi, University Hospital, Department of Psychiatry, Otto von Guericke University Magdeburg, Leipzigerstr. 44, Magdeburg 39120, Germany, Tel: +4915225191188, Fax: +4939167|4229, E-mail: leonardo.tozzi@med.ovgu.de

Received I August 2017; revised 15 October 2017; accepted 14 November 2017; accepted article preview online 28 November 2017
2009). In particular, increased intracellular levels of FKBP5 decrease the affinity of the glucocorticoid receptor (GR, Binder, 2009) after being upregulated by active corticosteroid receptors at the transcriptional level through GR response elements (GREs) (Wochnik et al, 2005; Grad and Picard, 2007). Compatible with its role in HPA axis regulation, variations of FKBP5 have been investigated in several conditions that involve exposure to chronic stress, such as gastric cancer (Kang et al, 2012), childhood maltreatment (Gillespie et al, 2009), post-traumatic stress disorder (Binder et al, 2008).

How chronic stress might influence FKBP5 gene transcription and its protein function is not known, but it has been suggested that their interplay might be mediated by epigenetic mechanisms (Tyrka et al, 2016). In support of this view, treatment of human hippocampal progenitor cells with glucocorticoids induced long-lasting demethylation of FKBP5 regulatory intronic regions and increased its expression (Klengel et al, 2013). Recent work has expanded this finding to human populations by showing that exposure to 
chronically high cortisol levels in Cushing syndrome was associated with lower DNA methylation level of FKBP5 introns assessed in white blood cells and smaller hippocampal volumes (Resmini et al, 2016). Decreased methylation was also observed in DNA taken from saliva samples of maltreated children (Tyrka et al, 2015) and in whole blood and saliva samples of adult victims of childhood trauma (Klengel et al, 2013). Therefore, current findings suggest that methylation levels at regulatory regions might constitute a link between FKBP5 expression and both endocrine as well as environmental stress.

MDD is a multifactorial condition with complex inheritance, that is likely to involve the interaction between genetic predisposition and environmental triggers, which result in persistent dysregulation of the HPA axis (Tafet and Nemeroff, 2016). Therefore, it is not surprising that variants of FKBP5 have been found to be associated with this disorder in several studies (see Rao et al (2016) for a recent metaanalysis). Furthermore, a role of FKBP5 in mediating antidepressant treatment response has been highlighted (Horstmann et al, 2010; Binder et al, 2004).

It is still unclear which role FKBP5 might play in the structural and functional brain differences detected between MDD patients and healthy controls (HC). Some magnetic resonance imaging (MRI) experiments have investigated gray matter (GM) volume, white matter integrity, and neural responses to stimuli in patients carrying high-risk allele variants of FKBP5 rs1360780 SNP relative to individuals without a risk allele, highlighting structural and functional differences in brain areas involved in emotional processing. For instance, in patients carrying the minor (risk) FKBP5 alleles, studies found volume changes in the amygdala and middle and inferior orbitofrontal gyri (Hirakawa et al, 2016) and dorsal anterior cingulate cortex (ACC) (Fujii et al, 2014). In these participants, white matter integrity was also found to be altered in the ACC (Fujii et al, 2014), insula, and inferior frontal gyrus (Tozzi et al, 2015). Functional studies, on the other hand, showed higher functional responses in the amygdala during an emotional face matching task which were also influenced by exposure to childhood trauma (White et al, 2012).

The first study investigating the interaction of MDD, FKBP5 allele status, methylation, and GM changes in depressed patients has only recently been conducted (Han et al, 2017). In this work, the $\mathrm{T}$ allele of $\mathrm{rs} 1360780$ was associated with volume reduction of portions of the frontal and parietal cortex, but exclusively in patients. Interestingly, an effect of FKBP5 methylation on right frontopolar gyrus GM thickness was detected in participants regardless of diagnosis, but dependent on rs1360780 allelic status. Overall, the authors provide further evidence that rs1360780 and MDD have interactive effects on GM volumes of cortical regions involved in emotion processing and mood regulation. In addition to this, FKBP5 methylation might predict changes in the structure of these areas depending on rs1360780.

Childhood adversity has been shown to interact with rs1360780 and induce FKBP5 demethylation (Binder et al, 2008; Klengel et al, 2013). Furthermore, in a previous study, we showed how MDD patients carrying the high-risk allele of rs1360780 showed reduced structural integrity and activation in the insula and inferior frontal gyrus depending on the amount of adversity endured (Tozzi et al, 2015). To our knowledge, the interaction between maltreatment, genetics, and epigenetics of FKBP5 on both brain structure and function has not yet been comprehensively explored in MDD, as highlighted by Han et al as a limitation of their study (Han et al, 2017).

Our goal was therefore to investigate, in MDD patients and $\mathrm{HC}$, the relationship between epigenetic modifications in FKBP5 with allelic status of rs1360780, exposure to childhood adversity and structural as well as functional brain measures. First, we investigated the methylation of FKBP5 CG-sites in whole blood DNA samples, expecting to find lower mean methylation levels in MDD patients expressing the $\mathrm{T}$ allele of rs1360780 and exposed to childhood abuse, compatibly with Klengel et al (2013). We then acquired structural MRI scans of our participants as well as functional ones during an emotion recognition task that consistently showed activation in emotional processing areas in the past (Tozzi et al, 2015, 2017). We hypothesized that lower whole blood FKBP5 methylation would also be correlated with lower GM content and activation in emotional processing areas (Klengel et al, 2013; Han et al, 2017). Finally, we assessed if the regions whose activity and GM concentration were correlated with FKBP5 methylation were also relevant for depression psychopathology.

\section{MATERIALS AND METHODS}

\section{Sample Composition}

We used data from two cohorts, which we collected independently between 2009 and 2015 at two imaging sites in Trinity College Dublin using the same Philips 3T MRI system: the Centre for Advanced Clinical Imaging (CAMI) and the Trinity College Institute of Neuroscience (TCIN). The data set comprised $53 \mathrm{HC}$ and $60 \mathrm{MDD}$.

Exclusion criteria were age $<18$ or $>65$, history of neurological or any comorbid psychiatric disorders including alcohol or substance dependency (Axis I), personality disorders (Axis II), other severe medical illness, psychosis, head injury or current substance abuse. Demographic variables, inclusion and exclusion criteria were documented by a psychiatrist using a standardized questionnaire.

Written informed consent was obtained from all participants after being given detailed description of the study which was designed and performed in accordance to the ethical standards laid out by the Declaration of Helsinki and was approved by the ethics committee of St. James and Tallaght hospitals, Dublin and McGill University, Montreal, Canada.

\section{Clinical Ratings}

The Hamilton Depression Scale (HAM-D) 21-item version (Hamilton, 1986), Beck's Depression Inventory (BDI) (BECK et al, 1961) as well as the Childhood Trauma Questionnaire (CTQ) (Bernstein and Fink, 1998) were filled out for all participants included in the study. The CTQ measures emotional, physical and sexual abuse as well as emotional and physical neglect. A three-item minimization-denial subscale is also included to check for attempts by respondents to minimize their childhood abuse experiences 
(Bernstein and Fink, 1998). The occurrence during his or her childhood of 28 items is rated by the participant on a 5-point Likert scale which ranges from 5 (no history of abuse or neglect) to 25 (extreme history of abuse or neglect). A total score is then obtained by adding all subscales (except the minimization-denial one).

\section{Epigenetic Analysis}

For a detailed description of the epigenetic analysis, see Supplementary Methods. Genomic DNA was extracted from whole blood samples, which had been collected into PAXgene Blood DNA Tubes. Since Resmini et al (2016) found more pronounced effects at CG-6 and CG-7 in the intron 7 region of FKBP5, we limited our analyses to these two sites, which are in or close proximity to a GRE (see supplementary figure 2 in Klengel et al, 2013).

Our region of interest (ROI) in the FKBP5 gene intron 7 was amplified by PCR. The following sequencing primers were used: FKBP5_S3A: 5'-ATTTTTGTGAAGGGTATAATT-3' and FKBP5-in7_S67: 5'-GTTGATATATAGGAATAAAATAAGA-3' (IDT Inc.) to assess CG-6 and CG-7 following the numbering of Resmini et al (2016), which correspond to Bin3, CG1-2 called by Klengel et al (2013). Finally, the average of CG-6 and CG-7 methylation was computed for each participant.

\section{rs1360780 Analysis}

For a detailed description of the rs1360780 analysis, see Supplementary Methods.

rs1360780 was genotyped from blood by the same laboratory for both sites using a Taqman SNP Genotyping Assay on a 7900HT Sequence Detection System (Applied Biosystems). Because homozygous TT samples were rare in our sample, we grouped them with heterozygous TC samples for analysis $\left(\mathrm{T}^{\star}\right)$ to define a binary rs1360780 variable.

\section{Demographic and Clinical Measures}

All statistical analyses were conducted using SPSS version 22 (IBM Corp).

Demographic variables and clinical test scores were compared between MDD and controls using independent $t$-tests (age, methylation percentage), chi-square tests (sex, rs1360780) and Mann-Whitney $U$ tests (CTQ, HAM-D, $\mathrm{BDI})$. The same tests were also conducted to compare participants based on the site and rs1360780 variables.

\section{Predictors of FKBP5 Methylation}

We entered methylation percentage as dependent variable in a full factorial general linear model (GLM) that had the following independent variables: diagnosis (binary: $\mathrm{HC}$ or MDD), site (binary: CAMI or TCIN), sex (binary: male or female), rs1360780 (binary: $\mathrm{CC}$ or $\mathrm{T}^{*}$ ), age (continuous), CTQ total score (continuous) as well as all four possible interactions between rs1360780 allele status, diagnosis, and CTQ total score. Upon identification of significant interactions, the post-hoc models were rerun splitting the data for the interacting factors. Within the MDD group, medication was added as a binary factor (medicated, unmedicated).

\section{Magnetic Resonance Imaging}

For a detailed description of MRI acquisition and analysis, see Supplementary Methods.

In summary, data were processed with Statistical Parametric Mapping 12 (SPM12, http://www.fil.ion.ucl.ac.uk/ spm) and with the Computational Anatomy Toolbox 12 (CAT12, http://www.neuro.uni-jena.de/vbm/download/).

Functional MRI task. A Neurobehavioral Systems Presentation (https://www.neurobs.com/) experiment was run during the fMRI recording as described in detail in our previous publications (Tozzi et al, 2017). The task was eventrelated and consisted of 180 pseudo-randomized trials, evenly distributed in 3 emotion $\times 2$ shape conditions. Each trial consisted of a fixation cross of jittered duration (mean $=1.5 \mathrm{~s}$, range: $1-1.8 \mathrm{~s}$ ), followed by positive, negative or neutral rectangular pictures from the International Affective Picture System database (IAPS) for $2 \mathrm{~s}$. After seeing the picture, participants were either asked to focus on the emotion elicited by the picture and answer whether this was positive, negative or neutral or had to answer a question about its shape (horizontal or vertical). From here on, we will label the first type of trial emotional recognition trials (ERT) and the second shape recognition trials (SRT). The same amount (30) of ERT and SRT was delivered for each of the three valences (positive, negative, neutral).

During pre-processing, movement parameters of all subjects were inspected and four patients and three controls were excluded from the sample, resulting in a final sample of $50 \mathrm{HC}$ and $56 \mathrm{MDD}$ patients. Structural data were segmented and warped to MNI space using the default CAT12 pipeline. Functional data were co-registered with the structural one and also normalized using the warp fields obtained during the normalization of the structural data (see Supplementary methods for details).

A first-level GLM analysis was conducted on the normalized functional data, using a canonical HRF as response function and a high-pass filter of $128 \mathrm{~s}$. Times at which the questions were presented were entered in the GLM along with the six motion parameters of each subject. T-tests were then run on ERT $>$ SRT first-level contrasts for each emotional valence (neutral, negative and positive), thus representing brain response to the evaluation of each emotion elicited by the pictures in comparison to that of their shape.

ROI definition and extraction. We used an ROI approach, targeting parts of the brain that are known to be involved in emotion recognition and to be especially affected in MDD, in particular the medial and lateral prefrontal cortex, amygdala, insula, and hippocampus (Phillips et al, 2008; Drevets et al, 2008).

The automated anatomical labeling atlas (Maldjian et al, 2003) as provided in the CAT12 toolbox was used to identify the following emotional processing structures (left and right): superior frontal gyrus, superior frontal orbital gyrus, middle frontal gyrus, middle frontal orbital gyrus, inferior frontal operculum, inferior frontal gyrus, inferior frontal orbital gyrus, superior medial frontal gyrus, medial frontal orbital gyrus, insula, anterior cingulum, hippocampus, and 
Table I Characteristics of Our Sample

\begin{tabular}{|c|c|c|c|c|c|}
\hline & \multicolumn{2}{|c|}{ CAMI } & \multicolumn{2}{|c|}{ TCIN } & \multirow[t]{2}{*}{ Test } \\
\hline & $\mathrm{HC}$ & MDD & $\mathrm{HC}$ & MDD & \\
\hline Age & $38.28(12.40)$ & $40.42(9.72)$ & $34.00(11.63)$ & $37.76(13.17)$ & $t=-1.20, p=0.23$ \\
\hline $\operatorname{Sex}(F / M)$ & $17 / 12$ & $21 / 10$ & $13 / 8$ & $15 / 10$ & Chi-square $=0.21, p=0.69$ \\
\hline HAMD & $2(0-7)$ & $29(17-45)$ & $0(0-5)$ & $23(6-3 \mid)$ & $U=2797.50, p<0.01$ \\
\hline BDI & $\mid(0-12)$ & $31(5-59)$ & I (0-13) & $34(|7-5|)$ & $U=2193, p<0.01$ \\
\hline CTQ total & $30(25-48)$ & $40(25-88)$ & $28(25-53)$ & $36(25-82)$ & $U=2084, p<0.01$ \\
\hline Medication type & & $9 / 12 / 4 / 3 / 3$ & & $4 / 14 / 3 / 2 / 2$ & \\
\hline
\end{tabular}

Abbreviations: BDI, Beck depression inventory; CAMI, Centre for Advanced Medical Imaging site; CTQ, childhood trauma questionnaire; HAMD, Hamilton scale for depression; HC, healthy controls; MDD, major depressive disorder patients; SNRI, serotonin-norepinephrine reuptake inhibitor; SSRI, selective serotonin reuptake inhibitor; TCIN, Trinity College Institute of Neuroscience site.

For our HC and MDD groups, descriptives of the following measures are given: age, sex, rs I 360780 allele status, peripheral mean intron 7 methylation percentage, clinical questionnaire scores, and medication. Parametric variables are shown as mean (SD), non-parametric ones as median (minimum-maximum). Medication is expressed as untreated/SSRI/SNRI/SSRI+SNRI/other (antipsychotic or agomelatine). Statistical tests for comparisons between MDD and HC and their results are also shown.

amygdala, for a total of 13 ROIs on each side (see Supplementary Table 1 for ROI details).

Mean activity change from the first level ERT $>$ SRT contrasts for each emotional valence, as well as mean GM concentration, were extracted for each of the ROIs. These values and estimated total intracranial volume (TIV) were then entered into SPSS Statistics version 22 (IBM Corp.) for statistical analysis.

Effect of FKBP5 methylation on brain structure and function. Generalized estimating equations (GEE) as implemented in SPSS were employed for subsequent analyses, setting an exchangeable working correlation matrix, linear scale response, and subject variable as the subject ID.

First, we ran a model for each of our 13 ROIs as follows. GM concentration was set as dependent variable (continuous). To limit the number of models in analyses, since we did not expect strongly lateralized effects of blood FKBP5 methylation percentage, we added the within-subject variable 'ROI side' (factor with two levels: left and right) to the model. Between-subjects independent variables were age, site (CAMI, TCIN), sex, rs1360780, diagnosis, FKBP5 methylation percentage, and TIV. All main effects as well as all possible interactions between FKBP5 methylation, diagnosis, and rs1360780 were tested using Wald Chi-Square tests, considering significant a $p<0.05$ false discovery rate (FDR) corrected for multiple comparisons (Benjamini, 2010).

We then assessed fMRI changes in our ROIs. First of all, to confirm that our ROIs showed significant BOLD activity in the experimental conditions, we conducted one-sample $t$ tests on their mean ERT $>$ SRT response across all participants and excluded the ROIs for which there was no significant activation from our analysis $(p>0.05)$. Then, we conducted another GEE analysis, defining activation as measured by our contrast values as dependent variable (continuous). Within-subject variables were ROI side and, since we did not expect correlates of blood FKBP5 methylation to be valence-specific, emotional valence (factor with three levels: neutral, negative and positive). Betweensubjects independent variables were site, age, sex, diagnosis, rs1360780, and FKBP5 methylation percentage. As before, all main effects as well as all possible interactions between FKBP5 methylation, diagnosis, and rs1360780 were tested.

Finally, since we found a main effect of diagnosis for inferior frontal gyrus pars orbitalis (IFGO) activation, we tested whether this was correlated to symptoms severity within the MDD group. To do so, we ran two Spearman correlations of the residuals from the GEE model (activation corrected for site, age, sex, medication, and rs1360780) with BDI total score and HAMD total score.

\section{RESULTS}

\section{Demographic and Clinical Measures}

Demographic information of our samples along with questionnaire scores and tests are summarized in Table 1. No differences were detected regarding mean age or sex and $\mathrm{T}$ allele distribution between patients and controls (all tests $p>0.05)$. Methylation of the FKBP5 intron 7 CG-6 and CG-7 sites were strongly correlated $(r=0.56, p<0.01)$.

Data from the two sites did not significantly differ for age, mean FKBP5 intron methylation, sex, rs1360780, CTQ, HAMD, and BDI. The same was true for $\mathrm{T}^{*}$ and CC participants (all tests $p>0.05$ ).

MDD showed higher CTQ scores compared to controls in both data sets (CAMI: $U=676.50, p=0.001$; TCIN: $U=387.5, p=0.006)$.

\section{Predictors of FKBP5 Methylation}

Our GLM analysis (see Supplementary Table 2 for model information) returned a significant effect for the triple interaction between diagnosis, rs1360780, and CTQ 


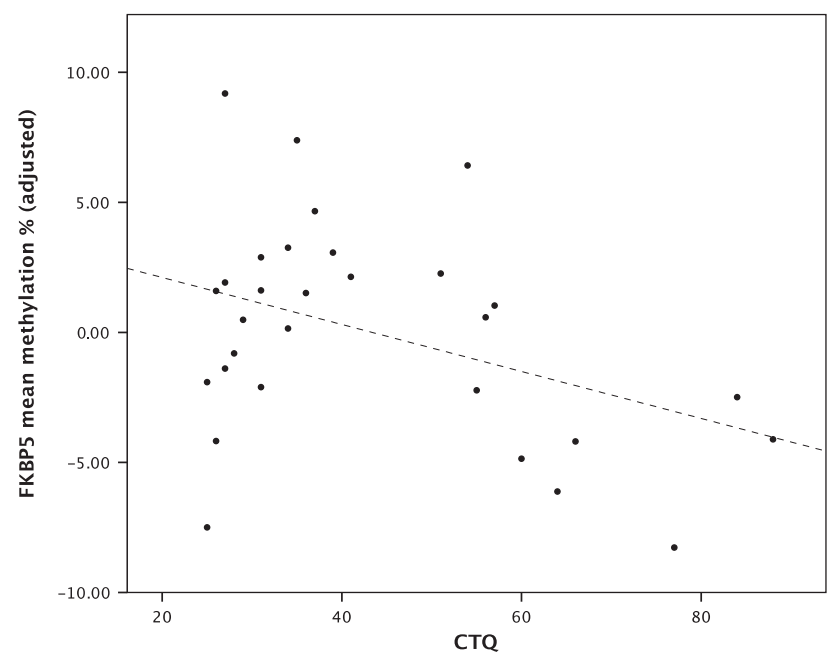

Figure I Correlation between peripheral mean FKBP5 methylation in T* patients and CTQ scores. Lower peripheral DNA methylation of FKBP5 intron 7 was associated to a higher exposure to childhood adversity in patients carrying the T allele of $r$ I $360780(F=4.95, p=0.04)$. Methylation values are adjusted for age, sex, site, and medication (residuals) and a leastsquares fit line is shown. CTQ, childhood trauma questionnaire.

$(\mathrm{F}=4.06, p=0.047)$. Post-hoc testing revealed a significant role for higher CTQ in predicting lower mean FKBP5 intron methylation in the $T^{\star}$ MDD group $(F=4.95, p=0.036$, Figure 1). Leverage values of the GLM were inspected: they were low, normally distributed (Kolmogorov-Smirnoff = $0.80, p=0.20$ ) and no outlier values (outside $1.5^{\star} \mathrm{IQR}$ ) in CTQ or FKBP5 were found. Residuals were also normally distributed (Kolmogorov-Smirnoff: 0.12, $p=0.20$ ).

\section{Effect of FKBP5 Methylation on Brain Structure and Function}

Our GEE models investigating the role of mean FKBP5 intron methylation in predicting GM concentration (see Supplementary Table 3 for models summary) returned a significant main effect for the IFGO across all participants (Wald chi-square $=11.93, p_{\text {FDR }}<0.01$, Figure 2 ).

All ROIs were significantly activated in the ERT $>$ SRT contrast across all participants across all valences (all $p<0.001$ ), with the exception of the inferior frontal gyrus pars opercularis $(t=-0.25, \quad p=0.80)$, insula $(t=0.36$, $p=0.72)$, middle frontal gyrus pars orbitalis $(t=-1.70$, $p=0.09)$ and superior frontal gyrus pars orbitalis $(t=-0.93$, $p=0.35$ ), which showed no significant functional response to our task and were therefore excluded from the analysis.

In the IFGO, we found a significant interaction between diagnosis and FKBP5 intron methylation in predicting functional responses (Wald chi-square $=6.57, p_{\mathrm{FDR}}=0.049$, see Supplementary Table 4 for models summary). To test whether this effect was valence-specific, we ran another model for this region including a methylation $\times$ valence term, as well as the main methylation effect. The interaction term was non-significant (chi $=0.318, p=0.853$ ) and the main effect of methylation remained significant (chi $=6.926$, $p=0.008$ ). Post-hoc investigation revealed that FKBP5 intron methylation showed a negative correlation with activation only in MDD regardless of valence and side (see

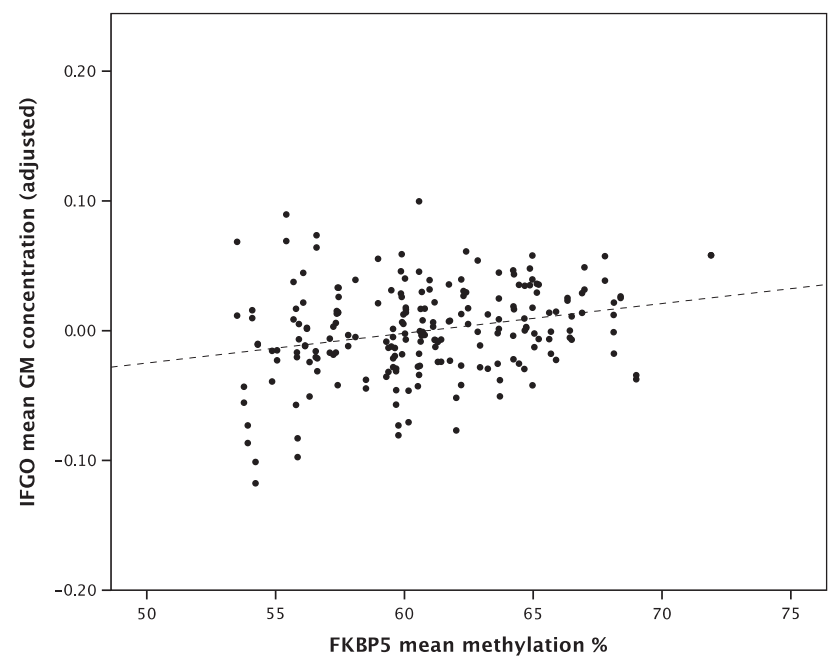

Figure 2 Correlation between IFGO mean GM concentration and peripheral mean FKBP5 methylation across all participants. Lower peripheral DNA methylation of FKBP5 intron 7 was associated to a lower mean gray matter concentration (Wald chi-square $=11.93, p_{\mathrm{FDR}}<0.0 \mathrm{I}$, to account for multiple regions). GM values are adjusted for site, age, TIV, diagnosis, sex, rs|360780 and side (residuals) and a least-squares fit line is shown. IFGO, inferior frontal gyrus pars orbitalis; GM, gray matter; TIV, total intracranial volume.

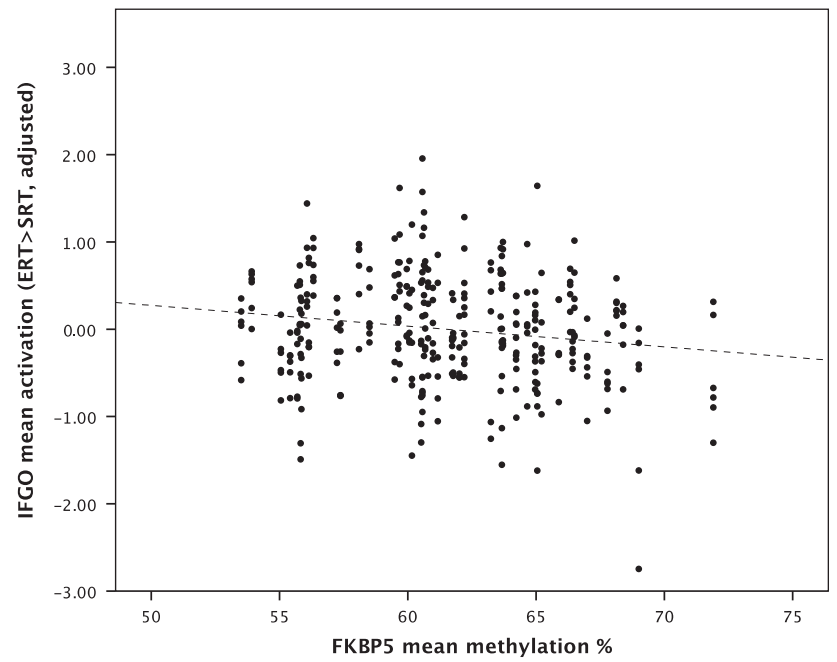

Figure 3 Correlation between IFGO mean activation and peripheral mean FKBP5 methylation in MDD. Lower peripheral DNA methylation of FKBP5 intron 7 was associated to a higher activation in ERT $>$ SRT in patients (Wald chi-square $=5.58, p=0.02$ ). Activation values are adjusted for site, age, sex, medication, rs/360780, valence and side (residuals) and a least-squares fit line is shown. IFGO, inferior frontal gyrus pars orbitalis; ERT, emotional recognition trials; SRT, shape recognition trials; MDD, major depressive disorder patients.

Supplementary Table 5 for model summary, Wald chisquare $=5.58, p=0.02$, Figure 3 ).

GM concentration in the IFGO was not different between MDD and HC (Wald chi-square $=0.37, p_{\mathrm{FDR}}=0.76$ ). However, its activation was lower in MDD compared to $\mathrm{HC}$ regardless of valence, age, side, and rs1360780 allele status (Wald chi-square $=3.88, p_{\mathrm{FDR}}=0.049$ ). Its activation was also significantly negatively correlated with BDI (Spearman's 
rho $=-0.143, p=0.009$ ) and HAMD (Spearman's rho $=$ $-0.179, p=0.002$ ) in MDD (corrected for site, age, sex, medication, and rs1360780).

\section{DISCUSSION}

Firstly, our study supports the hypothesis that, in the subpopulation of MDD patients carrying the high-risk $\mathrm{T}$ allele of rs 1360780 , lower methylation of FKBP5 introns is correlated to higher chronic stress exposure in early life. This finding is supported by numerous studies, ranging across several chronic stress conditions (Tyrka et al, 2016,2015; Provencal and Binder, 2015; Resmini et al, 2016; Klengel et al, 2013). We therefore expand to patients showing depressive symptoms the notion that FKBP5 demethylation is a correlate of exposure to childhood adversity, highlighting the role of rs1360780 as a moderator of this effect.

Furthermore, across all participants, lower FKBP5 intron methylation levels were associated with reduced GM concentration in the inferior frontal orbital gyrus. This region has been associated with response inhibition in general (Chikazoe et al, 2007) and, in particular, reappraisal and modulation of negative emotion (Goldin et al, 2008). Overall, structural changes in the orbitofrontal cortex in MDD are a common finding and have been confirmed at a meta-analytical level (Kempton et al, 2011). In rodents, exposure to chronic stress was associated with FKBP5 gene demethylation and hyper expression in the prefrontal cortex (Guidotti et al, 2013; Lee et al, 2010). Also, in a recent study comparing youths with post-traumatic stress disorder and controls, a negative association between GM in the orbitofrontal cortex and evening cortisol levels across all participants was shown, regardless of diagnosis (Carrion et al, 2010). Taken together with these previous findings, our results suggest that FKBP5 methylation might be related to GR function in chronic stress conditions and might influence the structural integrity of the inferior frontal orbital gyrus in both MDD and HC. Since cortisol measures were not available and childhood adversity was considerably more present in our depressed patients, we can provide supporting evidence for this theory only in our MDD $\mathrm{T}^{\star}$ group, in which FKBP5 intron demethylation was specifically explained by exposure to childhood adversity.

We found activation of the IFGO to be reduced in MDD patients compared to $\mathrm{HC}$, and to be inversely correlated with the self-reported intensity of symptoms. Interestingly, in MDD patients, FKBP5 methylation was negatively correlated with IFGO hemodynamic response during our emotional recognition task regardless of valence. This finding is surprising, since after observing that lower FKBP5 methylation was associated with lower GM in the IFGO, we expected it would rather mirror a reduction of function in this region. In addition, the lack of valence-specificity of the prediction of methylation on functional responses could hint at a change which affects the area's function in general. It is possible that the heterogeneity of our findings could reflect one of the underlying biological pathways leading to MDD. A subset of patients, for example, who could have developed it after enduring chronic stress exposure, might show lower FKBP5 methylation, less symptoms and higher functional responses to emotional evaluation. The consequence for the clinical outcome of these patients would be interesting to know, but our cross-sectional data do not allow us to investigate this aspect. On the other hand, HPA axis dysregulation has been associated with more severe depressive symptoms intensity in the past (Burke et al, 2005; Guidotti et al, 2013). Since the effect of medication approached significance in predicting higher activity in the IFGO $(p=0.07)$, another possibility might be that FKBP5 epigenetics could play a role in successful response to antidepressant therapy, although methylation was not different between treated and untreated patients in our sample, nor in Han et al (2017).

This study is not without limitations. First of all, it is necessary to address the fact that most of our patients were medicated and, although we corrected for medication use in all of our models, we cannot exclude that our results might be confounded by this factor. Secondly, we used data from two cohorts collected at two sites where MRI measurements were conducted using different sequences and receiver coils (although both sites used the same scanner model and, crucially, the TR for the functional acquisition was the same). Pooling data from different sites allowed us to increase our sample size and we tried to minimize differences between them by using the same processing pipeline and correcting for site in all our analyses. However, we cannot exclude that differences between sites might have confounded our results. Thirdly, our results differ from those reported by Han et al (2017) in a recent similar study. This might be due to several reasons, such as the use of different methods (VBM and fMRI compared to automated cortical segmentation using Freesurfer) and a different choice of ROIs and statistics. However, the present study addresses some of the limitations highlighted in Han et al (2017), for example the investigation of the role of childhood trauma in predicting FKBP5 intron methylation, which we consider valuable for the interpretation of our findings. Furthermore, both studies are in agreement in highlighting the inferior frontal gyrus as a crucial site for the role of FKBP5 on brain structure. Also, we did not have reliable measures of HPA axis function, such as cortisol levels. Another important point to be addressed is the still not well-known association between peripheral and central FKBP5 methylation. The results of the present study suggest that peripheral FKBP5 methylation is linked to CNS structure and function, but experimental research on this link is necessary. Finally, we did not detect any significant findings in the hippocampus. This region has been shown to be smaller in MDD (Schmaal et al, 2016; Frodl et al, 2002). Its volume was also positively correlated to FKBP5 methylation in Cushing's syndrome patients (Resmini et al, 2016) and its shape was different between PTSD carrying the T allele of rs1360780 (Fani et al, 2013). This might suggest that this structure does not show strong effects of FKBP5 regulation in MDD compared to other forms of stress exposure, such as Cushing's syndrome. The effects could also be weaker in this disorder and would need a larger sample size to be detected. To elucidate the matter, targeted studies should be conducted on this region, perhaps using dedicated segmentation methods to account for its structural complexity, such as those provided in the Freesurfer suite (https:// surfer.nmr.mgh.harvard.edu/).

Overall, future studies might benefit from the investigation of the interaction between known genetic variants of FKBP5 
and its methylation in large cohorts of medicated and unmedicated MDD patients, carefully controlling for childhood trauma levels, directly assessing HPA axis function and focusing on imaging the inferior frontal gyrus.

To summarize, in a subgroup of MDD patients carrying the high-risk allele of the FKBP5 rs1360780 SNP, exposure to childhood trauma was inversely correlated to peripheral DNA methylation level of this gene. Across all participants, lower FKBP5 methylation was associated with decreased GM in the inferior frontal orbital gyrus. In the MDD group, lower methylation correlated with its higher activation. This region is linked with emotional regulation and was functionally hypoactive in MDD in our sample, with its activation being negatively correlated with self-reported symptoms severity. Our findings suggest that epigenetic changes of FKBP5 might be a link connecting the interaction of genetic and environmental risks with brain changes in an area relevant for the clinical symptoms of depression.

\section{FUNDING AND DISCLOSURE}

Prof Thomas Frodl, Dr Leonardo Tozzi, and Dr Zsofia Nemoda report having received funding from the European Union through the Marie Curie Programme. Prof Veronica O'Keane received funding from The Meath Foundation, Dublin, Ireland for the HPA axis work. DNA methylation analyses were supported by a grant from the Fonds de Recherche Quebec-Santé awarded to Dr Linda Booij. Dr Linda Booij is supported by a New Investigator Award from the Canadian Institutes of Health Research (CIHR). Julian Chiarella is supported by a Frederick Banting and Charles Best Canada Graduate Scholarship from CIHR. All authors declare no conflict of interest.

\section{REFERENCES}

Beck AT, Ward CH, Mendelson MM, Mock JJ, Erbaugh JJ (1961). An inventory for measuring depression. Arch Gen Psychiatry 4: 561-571.

Benjamini Y (2010). Discovering the false discovery rate. $J$ R Stat Soc Ser B (Stat Methodol) 72: 405-416.

Bernstein DP, Fink L (1998). Childhood Trauma Questionnaire: A Retrospective Self-Report Manual. The Psychological Corporation: San Antonio, TX.

Binder EB (2009). The role of FKBP5, a co-chaperone of the glucocorticoid receptor in the pathogenesis and therapy of affective and anxiety disorders. Psychoneuroendocrinology 34 (Supplement 1 (December)): S186-S195.

Binder EB, Bradley RG, Liu W, Epstein MP, Deveau TC, Mercer KB et al (2008). Association of FKBP5 polymorphisms and childhood abuse with risk of posttraumatic stress disorder symptoms in adultsJAMA 299: 1291-1305.

Binder EB, Salyakina D, Lichtner P, Wochnik GM, Ising M, Pütz B et al (2004). Polymorphisms in FKBP5 are associated with increased recurrence of depressive episodes and rapid response to antidepressant treatment. Nat Genet 36: 1319-1325.

Burke HM, Davis MC, Otte C, Mohr DC (2005). Depression and cortisol responses to psychological stress: a meta-analysis. Psychoneuroendocrinology 30: 846-856.

Carrion VG, Weems CF, Richert K, Hoffman BC, Reiss AL (2010). Decreased prefrontal cortical volume associated with increased bedtime cortisol in traumatized youth. Biol Psychiatry 68: 491-493.
Chikazoe J, Konishi S, Asari T, Jimura K, Miyashita Y (2007). Activation of right inferior frontal gyrus during response inhibition across response modalities. J Cogn Neurosci 19: 69-80.

Drevets WC, Price JL, Furey ML (2008). Brain structural and functional abnormalities in mood disorders: implications for neurocircuitry models of depression. Brain Struct Func 213: 93-118.

Fani N, Gutman D, Tone EB, Almli L, Mercer KB, Davis J et al (2013). FKBP5 and attention bias for threat: associations with hippocampal function and shape. JAMA Psychiatry 70: 392-400.

Frodl T, Meisenzahl EM, Zetzsche T, Born C, Groll C, Jäger M et al (2002). Hippocampal changes in patients with a first episode of major depression. Am J Psychiatry 159: 1112-1118.

Fujii T, Ota M, Hori H, Hattori K, Teraishi T, Sasayama D et al (2014). Association between the common functional FKBP5 variant (rs1360780) and brain structure in a non-clinical population. J Psychiatr Res 58(November): 96-101.

Gillespie CF, Phifer J, Bradley B, Ressler KJ (2009). Risk and resilience: genetic and environmental influences on development of the stress response. Depress Anxiety 26: 984-992.

Goldin PR, McRae K, Ramel W, Gross JJ (2008). The neural bases of emotion regulation: reappraisal and suppression of negative emotion. Biol Psychiatry 63: 577-586.

Grad I, Picard D (2007). The glucocorticoid responses are shaped by molecular chaperones. Mol Cell Endocrinol 275: 2-12.

Guidotti G, Calabrese F, Anacker C, Racagni G, Pariante CM, Riva MA (2013). Glucocorticoid receptor and FKBP5 expression is altered following exposure to chronic stress: modulation by antidepressant treatment. Neuropsychopharmacology 38: 616-627.

Hamilton M (1986). The Hamilton Rating Scale for Depression. In: $\mathrm{N}$ Sartorius and TA Ban (eds). Assessment of Depression. Springer: Berlin Heidelberg, pp 143-152.

Han K-M, Won E, Sim Y, Kang J, Han C, Kim Y-K et al (2017). Influence of FKBP5 polymorphism and DNA methylation on structural changes of the brain in major depressive disorder. Sci Rep 7: 42621.

Hirakawa H, Akiyoshi J, Muronaga M, Tanaka Y, Ishitobi Y, Inoue A et al (2016). FKBP5 is associated with amygdala volume in the human brain and mood state: a voxel-based morphometry (VBM) study. Int J Psychiatry Clin Pract 20: 106-115.

Horstmann S, Lucae S, Menke A, Hennings JM, Ising M, Roeske D et al (2010). Polymorphisms in GRIK4, HTR2A, and FKBP5 show interactive effects in predicting remission to antidepressant treatment. Neuropsychopharmacology 35: 727-740.

Kang JI, Chung HC, Jeung H-C, Kim SJ, An SK, Namkoong K (2012). FKBP5 polymorphisms as vulnerability to anxiety and depression in patients with advanced gastric cancer: a controlled and prospective study. Psychoneuroendocrinology 37: 1569-1576.

Kempton MJ, Salvador Z, Munafò MR, Geddes JR, Simmons A, Frangou $S$ et al (2011). Structural neuroimaging studies in major depressive disorder. meta-analysis and comparison with bipolar disorder. Arch Gen Psychiatry 68: 675-690.

Klengel T, Mehta D, Anacker C, Rex-Haffner M, Pruessner JC, Pariante CM et al (2013). Allele-specific FKBP5 DNA demethylation mediates gene-childhood trauma interactions. Nat Neurosci 16: 33-41.

de Kloet ER, Joëls M, Holsboer F (2005). Stress and the brain: from adaptation to disease. Nat Rev Neurosci 6: 463-475.

Lee RS, Tamashiro KLK, Yang X, Purcell RH, Harvey A, Willour VL et al (2010). Chronic corticosterone exposure increases expression and decreases deoxyribonucleic acid methylation of Fkbp5 in mice. Endocrinology 151: 4332-4343.

Maldjian JA, Laurienti PJ, Kraft RA, Burdette JH (2003). An automated method for neuroanatomic and cytoarchitectonic atlas-based interrogation of fMRI data sets. NeuroImage 19: 1233-1239.

Miller AH, Raison CL (2016). The role of inflammation in depression: from evolutionary imperative to modern treatment target. Nat Rev Immunol 16: 22-34.

Phillips ML, Ladouceur CD, Drevets WC (2008). A neural model of voluntary and automatic emotion regulation: implications for 
understanding the pathophysiology and neurodevelopment of bipolar disorder. Mol Psychiatry 13: 829-857.

Provencal N, Binder EB (2015). The neurobiological effects of stress as contributors to psychiatric disorders: focus on epigenetics. Curr Opin Neurobiol 30(February): 31-37.

Rao S, Yao Y, Ryan J, Li T, Wang D, Zheng C et al (2016). Common variants in FKBP5 gene and major depressive disorder (MDD) susceptibility: a comprehensive meta-analysis. Sci Rep 6(September): 32687.

Resmini E, Santos A, Aulinas A, Webb SM, Vives-Gilabert Y, Cox O et al (2016). Reduced DNA methylation of FKBP5 in Cushing's Syndrome. Endocrine 54: 768-777.

Scharf SH, Liebl C, Binder EB, Schmidt MV, Müller MB (2011). Expression and regulation of the Fkbp5 gene in the adult mouse brain. PLoS One 6: e16883.

Schmaal L, Veltman DJ, van Erp TGM, Sämann PG, Frodl T, Jahanshad $N$ et al (2016). Subcortical brain alterations in major depressive disorder: findings from the ENIGMA Major Depressive Disorder Working Group. Mol Psychiatry 21: 806-812.

Tafet GE, Nemeroff CB (2016). The links between stress and depression: psychoneuroendocrinological, genetic, and environmental interactions. J Neuropsychiatry Clin Neurosci 28: 77-88.

Tozzi L, Carballedo A, Wetterling F, McCarthy H, O'Keane V, Gill $M$ et al (2015). Single-nucleotide polymorphism of the FKBP5 gene and childhood maltreatment as predictors of structural changes in brain areas involved in emotional processing in depression. Neuropsychopharmacology 41: 487-497.

Tozzi L, Doolin K, Farrel C, Joseph S, O’Keane V, Frodl T (2017). Functional magnetic resonance imaging correlates of emotion recognition and voluntary attentional regulation in depression: a generalized psycho-physiological interaction study. J Affect Disord 208: 535-544.

Tyrka AR, Parade SH, Eslinger NM, Marsit CJ, Lesseur C, Armstrong $\mathrm{DA}$ et al (2015). Methylation of exons $1 \mathrm{D}, 1 \mathrm{~F}$, and $1 \mathrm{H}$ of the glucocorticoid receptor gene promoter and exposure to adversity in preschool-aged children. Dev Psychopathol 27: 577-585.

Tyrka AR, Ridout KK, Parade SH (2016). Childhood adversity and epigenetic regulation of glucocorticoid signaling genes: associations in children and adults. Dev Psychopathol 28: 1319-1331.

White MG, Bogdan R, Fisher PM, Muñoz KE, Williamson DE, Hariri AR (2012). FKBP5 and emotional neglect interact to predict individual differences in amygdala reactivity: FKBP5, emotional neglect and amygdala reactivity. Genes Brain Behav 11: 869-878.

Wochnik GM, Rüegg J, Abel GA, Schmidt U, Holsboer F, Rein T (2005). FK506-binding proteins 51 and 52 differentially regulate dynein interaction and nuclear translocation of the glucocorticoid receptor in mammalian cells. J Biol Chem 280: 4609-4616. 